\title{
Esiste un cutoff alla penetranza della dialisi peritoneale? Come superarlo?
}

\author{
Massimo Sandrini, Valerio Vizzardi, Luigi Manili, Giovanni Cancarini
}

U.O. Nefrologia, Spedali Civili e Università di Brescia, Brescia

\begin{abstract}
Does Penetration of PERITONeAl dialysis haVe a CUTOFF? How to OVERCOME it?
Abstract. At the dialysis center of Brescia, with experience in peritoneal dialysis (PD) of more than 30 years, the incidence of PD is $35 \%$. Despite this, the prevalence of $P D$ has an apparently fixed cutoff at about 80 patients. This short paper synthesizes some possible solutions to increase penetration of PD.
\end{abstract}

Key words: Peritoneal dialysis, Incidence, Prevalence

Conflict of interest: None.

Financial support: None.

Accettato: 21 Luglio 2013

Per aderire all'invito di Marco Lombardi, abbiamo iniziato rivedendo, nel supplemento GTND 2001, l'intervento di Amedeo De Vecchi e le conclusioni del coordinatore sulle motivazioni del mancato incremento di utilizzo della DP (1). Ancora oggi, dopo 12 anni, valgono le considerazioni di allora; per questo motivo, anziché tornare su quelle, analizziamo i nostri dati (Tab. I) per individuare i principali ostacoli a un'ulteriore crescita della DP nel centro di Brescia.

Questi dati indicano che non sussistono bias da mancata esperienza, pregiudizio, scarse dimensioni del centro o mancata selezione e informazione dei pazienti uremici terminali. Recentemente, Blake (2) ha definito che un'incidenza in DP del $33 \%$ è indice di elevato uso della DP e di efficiente programma di selezione; il nostro centro raggiunge questo target, ma è come se avesse un cutoff alla penetranza della DP, che resiste nonostante un consistente aumento di pazienti incidenti (Tab. I).

Il cutoff di utilizzo della DP potrebbe essere innalzato superando alcune criticità, tra le quali la più importante è la miopia della politica sanitaria vigente: Regione, Aziende Sanitarie e Aziende Ospedaliere non sembrano capaci di organizzare una sintesi che consideri costo complessivo del trattamento del paziente uremico (materiale per dialisi, personale sanitario e non, strutture dedicate, spesa farmaceutica, ospedalizzazioni, trasporti, costi sociali) e capacità di raggiungere l'obiettivo primario di cura (cioè sopravvivenza, riabilitazione e qualità di vita del paziente). In un simile confronto, la DP è il tipo di dialisi a minor costo e a pari efficacia; investire parte del risparmio generato dal suo minor costo, per stimolare un suo maggiore utilizzo, è la soluzione razionale.
Considerando la crescente fragilità dei pazienti avviati alla dialisi e le caratteristiche degli attuali nuclei familiari, andrebbero diffuse iniziative come il contributo economico alle famiglie o la DP domiciliare assistita o video-assistita. Per i pazienti già istituzionalizzati in RSA andrebbe proposto un incentivo alle strutture disponibili a praticare la DP.

Fondamentali per una maggiore penetranza della DP sono: afferenza precoce al nefrologo, percorso informativo adeguatamente strutturato e disponibilità e conoscenza di tutte le opzioni di trattamento dell'IRC:

- trapianto da vivente o cadavere (singolo o doppio), preemptive o successivo

- HD in centro, CAL o domiciliare

- CAPD e APD, a domicilio o RSA

- Dialisi Peritoneale Intermittente (IPD) ospedaliera (indicazioni limitate)

- dieta vegetale addizionata con cheto-analoghi

- trattamento conservativo a oltranza (cure palliative)

Un singolo centro difficilmente può offrire tutte queste opzioni che, tuttavia, tutte, vanno garantite al paziente; va, quindi, costituita una rete fra le diverse realtà nefrologiche.

Altre considerazioni sul percorso pre-dialitico:

a) per caratteristiche cliniche e culturali, l'equipe della DP è particolarmente adatta per attuare il percorso clinico-educazionale dedicato alla malattia renale avanzata;

b) un percorso pre-dialitico strutturato, oltre a ridurre la morbilità e ad aumentare la sopravvivenza dei pazienti dopo l'inizio della terapia sostitutiva, ritarda sensibilmente l'ingresso in dialisi; 
TABELLA I - CARATTERISTICHE DEL CENTRO DI DP DI BRESCIA (SITUAZIONE AL DICEMBRE DEL 2012)

\begin{tabular}{|c|c|c|}
\hline & & Note \\
\hline Inizio programma di DP & 1979 & \multirow{6}{*}{ (censimento GSPD 2010: 17\%) (2) } \\
\hline Pazienti trattati & 896 & \\
\hline Prevalenza DP/totale dializzati & $22 \%$ & \\
\hline Incidenza DP/totale incidenti & $35 \%$ & \\
\hline Anno di attivazione dell'ambulatorio Malattia Renale Avanzata (MaReA) & 2005 & \\
\hline Incidenza DP tra i pazienti MaReA senza controindicazioni cliniche alla DP & $41 \%$ & \\
\hline \multicolumn{3}{|l|}{ Confronto 2003 vs. 2012} \\
\hline 2003: incidenti/prevalenti & $22 / 79$ & \multirow{2}{*}{ A pari prevalenza, aumento del $50 \%$ di incidenza } \\
\hline 2012: incidenti/prevalenti & $33 / 79$ & \\
\hline
\end{tabular}

c) sono fondamentali i rapporti fra nefrologo e $\mathrm{MMG}$; vedasi il recente documento della Rete Nefrologia (ReNe) Lombarda (4);

d) in un'epoca di ospedali per acuti, vanno valorizzati i principi del "Chronic Care Model".

Per quanto riguarda l'inizio del trattamento dialitico, la DP incrementale ha permesso di aumentare la percentuale di pazienti incidenti in DP; pur in assenza di evidenze definitive a suo favore, il suo utilizzo è in progressivo aumento e ha raggiunto il 23\% nel 2010 (2).

Una menzione particolare merita il catetere peritoneale; nella nostra esperienza e nel censimento GSPD 2010 (2) esiste un dropout del $9 \%$ dei pazienti dovuto a problemi del catetere. Non è noto, inoltre, il numero di pazienti non avviati alla DP per impossibilità a posizionarlo o per criteri chirurgici eccessivamente restrittivi, forse da inesperienza. Un programma adeguato di DP richiede tempestività, competenza ed efficacia nel posizionamento del catetere; anche in questo caso, non è realistico pensare che tutti i centri rispondano in modo autonomo e adeguato. Un modello auspicabile dovrebbe comprendere nefrologi competenti per posizionamento chirurgico standard e chirurghi per interventi di secondo livello (anche in laparoscopia), inseriti in una rete organica (4).

Per completezza, non si può non accennare alla peritonite sclerosante (EPS) (5), complicanza che influenza la diffusione della metodica, prevalentemente in termini di pregiudizio, portando ad affermazioni come "metodica dialitica tempolimitata" o come "relativa controindicazione al trapianto". I punti critici relativi all'EPS sono:

- individuarne la patogenesi

- utilizzare esclusivamente soluzioni a maggiore biocompatibilità

- monitorare la funzione peritoneale

- individuare i segni precoci

- ottimizzare la terapia
- definire schemi di immunosoppressione post-trapianto per i pazienti DP

- pochi centri chirurgici di riferimento a livello nazionale.

Queste poche righe dovrebbero lasciar trasparire un autentico interesse per la DP e un appassionato tentativo di ottimizzarla e diffonderla, per migliorare la cura del paziente dializzato, gli stessi obiettivi che aveva Amedeo.

\section{Riassunto}

Il centro di Brescia ha un'incidenza della DP del 35\% e oltre 30 anni di esperienza. Nonostante ciò, ha un cutoff di prevalenza a 80 pazienti, che non riesce a superare. Questo breve scritto sintetizza alcune possibili soluzioni per incrementare l'utilizzo della DP.

Parole chiave: Dialisi peritoneale, Incidenza, Prevalenza

Dichiarazione di conflitto di interessi: Gli Autori dichiarano di non avere conflitto di interessi.

Contributi economici degli autori: Gli Autori dichiarano di non aver ricevuto sponsorizzazioni economiche per la preparazione dell'articolo.

Indirizzo degli Autori:

Prof. Giovanni Cancarini

U.S. Dialisi Peritoneale - U.O. Nefrologia

Spedali Civili di Brescia

Piazzale Spedali Civili 1

25123 Brescia

giovanni.cancarini@med.unibs.it 


\section{Bibliografia}

1. De Vecchi AF, Scalamogna A, Finazzi S, et al. Preliminary evaluation of incremental peritoneal dialisi in 25 patients. Perit Dial Int 2000; 20: 412-7.

2. Blake PG, Quinn RR, Oliver MJ. Peritoneal dialysis and the process of modality selection. Perit Dial Int 2013; 33: 233-41.

3. Marinangeli G, Cabiddu G, Neri L, et al. Old and New Perspectives on Peritoneal Dialysis in Italy Emerging from the Peritoneal Dialysis Study Group Census. Perit Dial Int 2012; 32: 558-65.
4. Decreto Direzione Generale Sanità Regione Lombardia; ultimo accesso al sito: 15/07/2013; http:/www.sanita.regione.lombardia.it/shared/ccurl/354/32/Decreto\%20approvazione\%20documenti\%20sottogruppi. PDF 96-100.

5. Peritonite Sclerosante Incapsulante (EPS), 2013. Estensore: Guido Garosi (UOC Nefrologia, Dialisi e Trapianto - Azienda Ospedaliera Universitaria Senese); ultimo accesso: 15/07/2013; http://www.nephromeet.com. 